\title{
MicroRNA-566 modulates vascular endothelial growth factor by targeting Von Hippel-Landau in human glioblastoma in vitro and in vivo
}

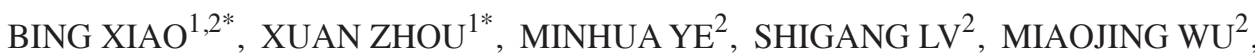 \\ CHANGCHUN LIAO ${ }^{2}$, LEI HAN ${ }^{3}$, CHUNSHENG KANG $^{3}$ and XINGEN ZHU ${ }^{2}$
}

\begin{abstract}
${ }^{1}$ Department of Maxillary Facial and Otorhinolaryngology Head \& Neck Surgery, Tianjin Medical University Cancer Institute \& Hospital, National Clinical Research Center for Cancer, Tianjin Key Laboratory of Cancer Prevention and Therapy, Tianjin 300060; ${ }^{2}$ Department of Neurosurgery, Second Affiliated Hospital of Nanchang University, Nanchang, Jiangxi 330006;

${ }^{3}$ Department of Neurosurgery, Tianjin Medical University General Hospital, Laboratory of Neuro-Oncology, Tianjin 300052, P.R. China
\end{abstract}

Received December 24,2014; Accepted September 22, 2015

DOI: $10.3892 / \mathrm{mmr} .2015 .4537$

\begin{abstract}
MicroRNAs (miRNAs) are able to function as either oncogenes or tumor suppressor genes in tumorigenesis, and have been proposed as novel targets for anticancer treatment. It has previously been suggested that miRNAs have important roles in the initiation and progression of glioblastoma; however, the effects of miR-566 in glioblastoma are currently unclear. The present study aimed to demonstrate that miR-566 can modulate vascular endothelial growth factor (VEGF) by targeting Von Hippel-Lindau (VHL) in glioblastoma in vitro and in vivo by inhibiting the expression of miR-566. Glioblastoma is a highly vascularized tumor, which exhibits increased expression of angiogenic factors, including VEGF, which are crucial in the process of glioblastoma angiogenesis. Existing research has demonstrated that VHL is a tumor suppressor gene that is associated with various tumors. In addition, VHL is able to regulate the expression of VEGF by promoting the degradation of hypoxia-inducible factor- $1 \alpha$ via ubiquitination. It has been predicted, using bioinformatics,
\end{abstract}

Correspondence to: Dr Xuan Zhou, Department of Maxillary Facial and Otorhinolaryngology Head \& Neck Surgery, Tianjin Medical University Cancer Institute \& Hospital, National Clinical Research Center for Cancer, Tianjin Key Laboratory of Cancer Prevention and Therapy, 2 Huanhuxi Road, Tianjin 300060, P.R. China

E-mail: byron2000zhou@sina.com

Professor Xingen Zhu, Department of Neurosurgery, Second Affiliated Hospital of Nanchang University, 1 Minde Road, Nanchang, Jiangxi 330006, P.R. China

E-mail: zxg2008vip@163.com

*Contributed equally

Key words: microRNA-566, Von Hippel-Landau, hypoxia-inducible factor-1 $\alpha$, vascular endothelial growth factor, glioblastoma that the VHL gene is regulated by miR-566. Therefore, the present study hypothesized that miR-566 may regulate VEGF expression by targeting VHL during the angiogenic process of glioblastoma multiforme. The results of the present study demonstrated that inhibition of miR-566 expression increases the expression levels of VHL, decreases the expression levels of VEGF, and inhibits the invasive and migratory abilities of glioblastoma. In addition, VHL was identified as a functional target of miR-566.

\section{Introduction}

Glioblastoma is the most common type of malignant tumor in the human central nervous system, and the median survival of patients with glioblastoma is $<1$ year (1). There are numerous therapeutic options for the treatment of glioblastoma, including surgery, radiation therapy and chemotherapy; however, the overall curative effect is poor $(2,3)$. Therefore, it is critical to explore the molecular mechanisms of glioblastoma, in order to produce more optimized and effective treatment strategies (4). Glioblastoma tumors are rich in blood vessels (5), and vascular endothelial growth factor (VEGF) has previously been reported to be overexpressed in glioblastomas (6). In recent years, targeting angiogenesis has been considered as a novel direction for cancer treatment. Therefore, research is currently focused on therapies that target VEGF. Monoclonal antibodies against VEGF and its receptor (7), ribozymes (8), VEGF antisense therapy (9) and vascular endothelial growth inhibitors (10), have all been used to treat glioblastoma, in order to inhibit angiogenesis.

MicroRNAs (miRNAs) are a class of short non-coding RNAs that control the expression of numerous genes (11). A previous study demonstrated that miRNAs are involved in tumor initiation and progression, and may function as tumor suppressors or oncogenes (12). miRNAs have an important role in the initiation and progression of glioblastoma (13), and regulate the proliferation, invasion and apoptosis of glioblastoma cells $(14,15)$. A previous study reported that the expression of 
miR-566, alongside four other miRNAs (miR-181d, miR-518b, miR-524-5p and miR-1227), is correlated with the prognosis of glioblastoma (16). Therefore, it has been suggested that investigation of miRNAs involved in glioblastoma may aid in the development of novel treatment strategies. In recent years, numerous studies regarding miRNAs have been conducted, and miRNAs have been identified as exhibiting an important role in the initiation and progression of cancer, and thus have been suggested as a target for the treatment of glioblastoma $(17,18-20)$.

VHL functions as a tumor suppressor gene and is located on chromosome 3p25 26, where it encodes a 213 amino acid protein, known as pVHL. Mutations in VHL have been shown to contribute to tumorigenesis (21), and VHL is also involved in the initiation and progression of glioma (22). Hypoxia-inducible factor (HIF)-1 $\alpha$ is a distinguished target of the E3 ligase activity of VHL, which targets HIF-1 $\alpha$ for degradation (23). In addition, the expression of VEGF is regulated by HIF- $1 \alpha$, and VEGF is considered the most important vascular growth factor in the initiation and progression of glioma (24).

At present, the function of miR-566 in glioma remains to be elucidated, and further research is required regarding the molecular mechanism underlying the pathogenesis of glioma. In the present study, VHL was identified as a functional target of miR-566, and was shown to regulate the expression of VEGF by targeting HIF- $1 \alpha$ for degradation. In addition, miR-566 was demonstrated to modulate VEGF by targeting VHL in vitro and vivo, and the inhibition of miR-566 expression was able to inhibit the invasion and migration of glioblastoma.

\section{Materials and methods}

Cell culture and reagents. The U87 human glioblastoma cell line was obtained from the Basic Medical Institute, Chinese Academy Of Medical Sciences (Beijing, China). The cells were cultured in Dulbecco's modified Eagle's medium (DMEM; Hyclone, Logan, UT, USA) supplemented with $10 \%$ heat-inactivated fetal bovine serum (FBS; Hyclone) at $37^{\circ} \mathrm{C}$ in an atmosphere containing $5 \% \mathrm{CO}_{2}$.

Lentiviral infection and gene transfection. Lentiviruses including an miR-566 inhibitor segment (lenti-AS-566) and a negative control segment (lenti-NC) were obtained from Shanghai GenePharma Co., Ltd. (Shanghai, China). The U87 glioblastoma cells were infected with lenti-AS-566 or lenti-NC, or transfected with a VHL negative control (VHL-NC), which was provided by Professor Jinquan Cheng (H. Lee Moffitt Cancer Center and Research Institute, Tampa, FL, USA) using Lipofectamine 2000 (Invitrogen; Thermo Fisher Scientific, Inc., Waltham, MA, USA) according to the manufacturer's protocol. The U87 cells were seeded in a 6 -well plate at $40 \%$ confluence with $4.8 \times 10^{5}$ cells/well. After $24 \mathrm{~h}$, cells were transfected in serum-free medium $(250 \mu \mathrm{l})$ with $5 \mu \mathrm{l}$ virus or $5 \mu \mathrm{l}$ plasmids using $5 \mu \mathrm{l}$ Lipofectamine 2000. After 4-6 h, medium was replaced with DMEM containing $10 \%$ FBS, and cells were incubated at $37^{\circ} \mathrm{C}$ in $5 \% \mathrm{CO}_{2}$.

$R N A$ extraction and reverse transcription-quantitative polymerase chain reaction ( $R T-q P C R)$ analysis. Total RNA was extracted from the cells using TRIzol (Invitrogen) $48 \mathrm{~h}$ post-infection/transfection. RT-qPCR was used to detect the expression levels of miR-566 and VEGF mRNA. A one-step RT-PCR kit (Takara Bio, Inc., Otsu, Japan) was used according to the manufacturer's protocol. RT-PCR of miRNA-566 was performed with a $200 \mathrm{ng}$ sample on a thermocycler (PTC-200; Bio-Rad, Hercules, CA, USA), the reaction conditions were as follows: $16^{\circ} \mathrm{C}$ for $30 \mathrm{~min} ; 42^{\circ} \mathrm{C}$ for $30 \mathrm{~min} ; 85^{\circ} \mathrm{C}$ for $10 \mathrm{~min}$. qPCR of miRNA-566 was performed with $2 \mu \mathrm{l}$ miRNA RT product on thermocycler (PTC-200; Bio-Rad), the reaction conditions were as follows: $95^{\circ} \mathrm{C}$ for $3 \mathrm{~min}, 95^{\circ} \mathrm{C}$ for $12 \mathrm{sec}$ and $62^{\circ} \mathrm{C}$ for $30 \mathrm{sec}$, for 40 cycles. RT-PCR of VEGF was performed with $5 \mu \mathrm{g}$ sample reaction conditions were as follows: $37^{\circ} \mathrm{C}$ for $5 \mathrm{~min}, 42^{\circ} \mathrm{C}$ for $60 \mathrm{~min}$, and $70^{\circ} \mathrm{C}$ for $10 \mathrm{~min}$. qPCR of VEGF was performed with $5 \mu$ l VEGF RT product, reaction conditions were as follows: $93^{\circ} \mathrm{C}$ for $1 \mathrm{~min}, 55^{\circ} \mathrm{C}$ for $1 \mathrm{~min}$, and $72^{\circ} \mathrm{C}$ for $1 \mathrm{~min}$, for 40 cycles). The following primers were used and were obtained from GenePharma Co., Ltd.: Forward: 5'-GGGCGCCUGUGAUCCCAAC-3' and reverse: 5'-UUCGCAGACGACGGGGUCG-5' for miRNA-566; and forward: 5'-ATCTTCAAGCCATCCTGTGTGC-3' and reverse: 5'-CTTTTAGGGACACCCGGAAC-3' for VEGF. The expression levels of mature miR-566 and VEGF were quantified using the $\Delta \Delta \mathrm{C}_{\mathrm{q}}$ method. Glyceraldehyde 3-phosphate dehydrogenase (GAPDH) served as the internal control, and the relative abundance of miRNA was normalized to U6 small nuclear RNA.

Protein extraction, western blot analysis and luciferase reporter assay. Protein samples were extracted using radioimmunoprecipitation assay buffer (Pierce Biotechnology, Inc., Rockford, IL, USA) and the protease inhibitor phenylmethylsulfonyl fluoride (Sigma-Aldrich, St. Louis, MO, USA) $24 \mathrm{~h}$ post-infection/transfection. The protein from each sample was separated by sodium dodecyl sulfate-polyacrylamide gel electrophoresis (5\% spacer gel and 10\% separation gel; Bio-Rad) and transferred to polyvinylidene fluoride membranes (EMD Millipore, Billerica, MA, USA). The membranes were then washed three times with Tris-buffered saline containing Tween (Amresco LLC, Solon, OH, USA), incubated in blocking buffer (Tiangen Biotech, Co., Ltd., Beijing, China) for $1 \mathrm{~h}$ at $37^{\circ} \mathrm{C}$, and incubated with primary antibodies and homologous secondary antibodies. The antibodies used were as follows: Mouse anti-GAPDH monoclonal antibody (1:1,000; TA-08; ZSGB-BIO, Beijing, China); rabbit antihuman VHL polyclonal antibody (1:100; ab135576; Abcam, Cambridge, UK); rabbit anti-human polyclonal HIF-1 $\alpha$ antibody $(1: 1,000 ; 21691-1$; SAB, College Park, MD, USA); rabbit anti-human VEGF polyclonal antibody $(1: 1,000$; sc-152; Santa Cruz Biotechnology Inc., Santa Cruz, CA, USA); mouse anti-human matrix metalloproteinase (MMP)-2 polyclonal antibody, (1:1,000; sc-53630; Santa Cruz Biotechnology Inc.); and mouse anti-human MMP-9 polyclonal antibody $(1: 1,000$; sc-13520; Santa Cruz Biotechnology Inc.). Horseradish peroxidase-labeled goat anti-rabbit (ZDR-5306, 1:1,000, ZSGB-BIO) or goat anti-mouse (ZDR-5307, 1:1,000, ZSGB-BIO) were used as secondary antibodies. The blots were visualized using enhanced chemiluminescence (W1001, Promega Corporation, Fitchburg, WI, USA) and a G:BOX F3 system (Syngene, Cambridge, UK). The bands were quantified using ImageJ software (1.48V, National Institutes of Health, Bethesda, 

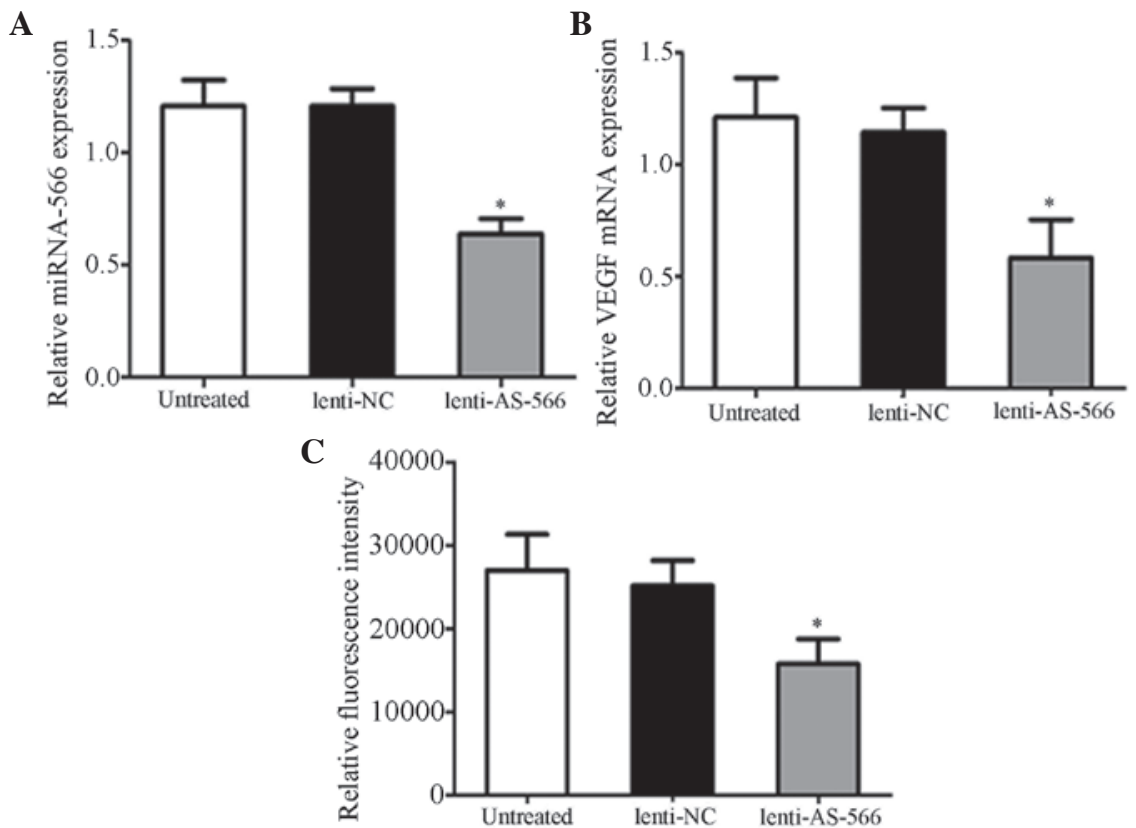

Figure 1. Downregulation of microRNA (miR)-566 inhibits the expression of vascular endothelial growth factor (VEGF) and the luciferase activity of Von Hippel-Landau (VHL). The expression levels of (A) miR-566 and (B) VEGF mRNA were significantly lower in the lentivirus (lenti)-AS-miR-566 (566)-infected cells, as compared with the untreated cells or those infected with lenti-normal control (NC), as determined by reverse transcription-quantitative polymerase chain reaction. These results indicated that lenti-AS-566 was able to effectively inhibit the expression of miR-566, and VEGF mRNA was downregulated by the suppression of miR-566. (C) VHL luciferase activity was significantly reduced in the lenti-AS-566-infected cells, as compared with the untreated cells or those infected with lenti-NC. These results indicated that the VHL gene is a direct target of miR-566. Data are presented as the mean \pm standard deviation. ${ }^{*} \mathrm{P}<0.05$, as compared with the untreated control group.

MD, USA). For the luciferase reporter assay, the cells were cultured in 96-well plates $(10,000$ cells/well), infected with $5 \mu$ lenti-AS-566 or lenti-NC, and incubated with $2 \mu 1$ Lipofectamine 2000 and $0.2 \mu \mathrm{g}$ pVHL luciferase reporter vectors. Following a $48 \mathrm{~h}$ incubation, luciferase activity was measured using a Dual-Luciferase Reporter system (Promega Corporation, Madison, WI, USA). Experiments were repeated at least three times.

Intracranial model. Female nude mice (age, 4-6 weeks; weight, 15-18 g; n=10/group) were bred at the Institute of Hematology (Tianjin, China). Mice were maintained in individually ventilated cages under a $12 \mathrm{~h}$ light/dark cycle, at $20-22^{\circ} \mathrm{C}$ and $40-60 \%$ humidity with free access to food and water. The mice were intracranially implanted with U87 cells infected with either lenti-AS-566 or lenti-NC under the direction of a stereotaxic apparatus (25). Briefly, mice were anesthetized by intraperitoneal injection of $10 \%$ chloral hydrate (MB1699, Meilunbio Company, Dalian, China). The mice were fixed on the brain stereotactic apparatus, and an incision was made to expose the brain, $5 \times 10^{5} / 3 \mu \mathrm{l}$ cells were injected and the skin was sutured. A total of 14 days post-cell implantation, the mice were sacrificed by $\mathrm{CO}_{2}$, and the brain tissue of the mice was collected, embedded in paraffin and cut into slices. All animal experiments were approved by the Institutional Animal Care and Use Committee (Tianjin Medical University Cancer Institute \& Hospital, Tianjin, China).

Fluorescence in situ hybridization and immunohistochemistry. The brain sections were routinely dewaxed and fixed in paraformaldehyde (Shanghai Bluestar New Chemical Materials Co., Ltd., Shanghai, China), and then digested with pepsin (Amresco LLC). The samples were subsequently incubated in prehybridization solution (Amresco LLC) for $4 \mathrm{~h}$ at $38^{\circ} \mathrm{C}$. Hybridization was performed in miR-566 hybridization probe liquid overnight at $38^{\circ} \mathrm{C}$. The samples were washed fully using sodium citrate-hydrochloric acid buffer solution (Leagene, Beijing, China), and incubated with mouse anti-digoxin antibody (Jackson ImmunoResearch Laboratories, Inc., West Grove, PA, USA) at $38^{\circ} \mathrm{C}$ for $2 \mathrm{~h}$, and $\mathrm{Cy} 3$ fluorescent antibody (Wuhan Boster Biological Technology, Ltd., Wuhan, China) at $38^{\circ} \mathrm{C}$ for $30 \mathrm{~min}$. The results were observed under a FV-1000 confocal laser-scanning microscope (Olympus Corporation, Tokyo, Japan).

Tissue sections were routinely deparaffinized and antigen retrieval was performed in $1 \mathrm{mM}$ EDTA buffer $(\mathrm{pH}$ 8.0) for $10 \mathrm{~min}$ at $95-100^{\circ} \mathrm{C}$. The sections were rinsed three times in phosphate-buffered saline (PBS) and then blocked in PBS containing 3\% normal horse serum for $30 \mathrm{~min}$. Subsequently, the sections were incubated with anti-VHL, -VEGF, -MMP-2 and -MMP-9 primary antibodies overnight (dilution, 1:100) at room temperature, rinsed in PBS and incubated with a goat anti-rabbit (sc-2040, 1:100, Santa Cruz Biotechnology, Inc.) or goat anti-mouse (sc-2039, 1;100, Santa Cruz Biotechnology, Inc.) biotinylated secondary antibody at $37^{\circ} \mathrm{C}$ for $40 \mathrm{~min}$ and $\mathrm{ABC}$-peroxidase reagents (Santa Cruz Biotechnology, Inc.) at $37^{\circ} \mathrm{C}$ for $1 \mathrm{~h}$. Immunohistochemical staining was performed using a DAB horseradish peroxidase color development kit (Beyotime Institute of Biotechnology, Shanghai, China) and evaluated under a CX21BIM-SET6 light microscope (Olympus Corporation). 
A
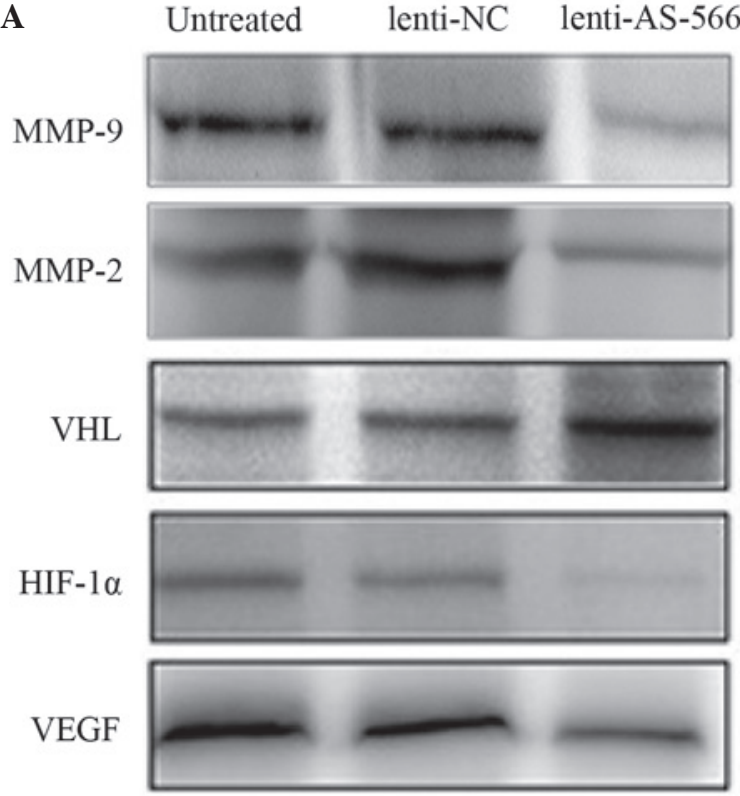

GAPDH

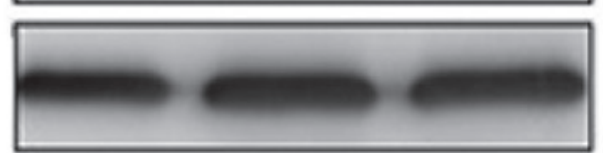

B

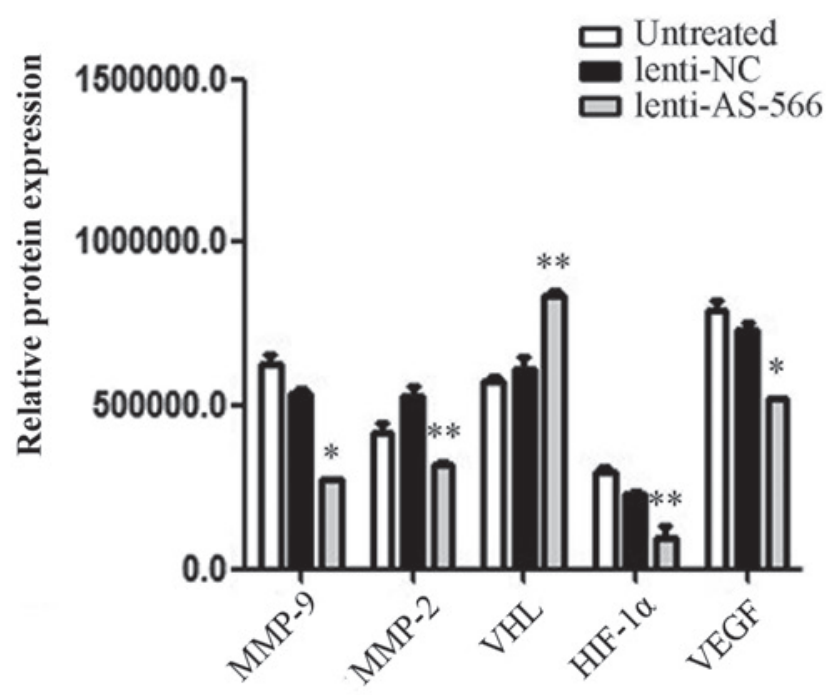

Figure 2. Relative protein expression levels in U87 cells following infection with lentivirus (lenti)-AS-microRNA-566 (566). (A) Western blot analysis demonstrated that the protein expression levels of Von Hippel-Landau (VHL) were increased, and hypoxia-inducible factor (HIF)-1 $\alpha$, vascular endothelial growth factor (VEGF), matrix metalloproteinase (MMP)-2 and MMP-9 were inhibited by the downregulation of microRNA-566. (B) Quantitative analysis of the western blotting results. Data are presented as the mean \pm standard deviation. ${ }^{*} \mathrm{P}<0.05$; ${ }^{* *} \mathrm{P}<0.01$, as compared with the untreated control group. GAPDH, glyceraldehyde 3-phosphate dehydrogenase.

A

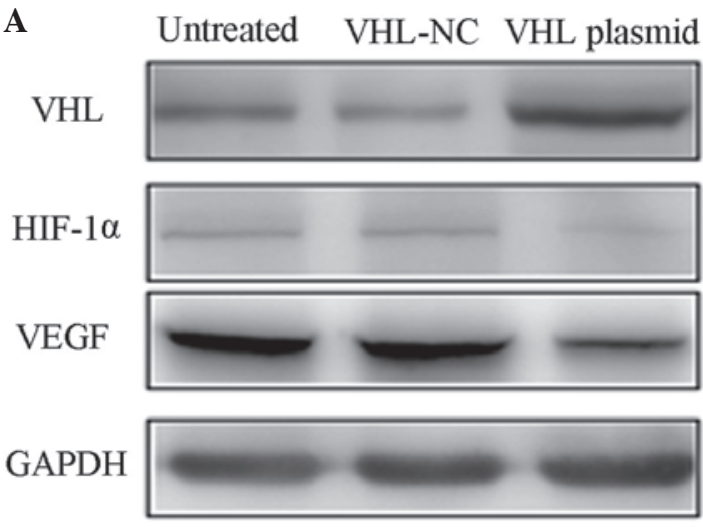

B

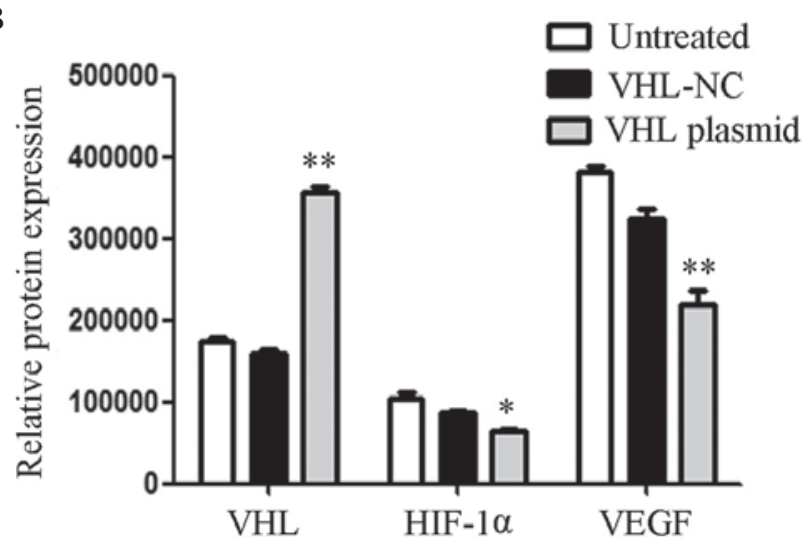

Figure 3. Relative protein expression levels in U87 cells following transfection with a Von Hippel-Landau (VHL) expression plasmid. (A) Western blot analysis revealed that the protein expression levels of VHL were increased, whereas hypoxia-inducible factor (HIF)-1 $\alpha$ and vascular endothelial growth factor (VEGF) were inhibited by the upregulation of VHL. (B) Quantitative analysis of the western blotting results. Data are presented as the mean \pm standard deviation. ${ }^{*} \mathrm{P}<0.05$ and ${ }^{* *} \mathrm{P}<0.01$, as compared with the untreated control group. NC, normal control; GAPDH, glyceraldehyde 3-phosphate dehydrogenase.

Statistical analysis. Data were statistically analyzed by $\chi^{2}$-test and one way analysis of variance, using SPSS 11.5 software (SPSS Inc., Chicago, IL, USA). P<0.05 was considered to indicate a statistically significant difference.

\section{Results}

Downregulation of miR-566 inhibits VEGF and miR-566 targets VHL. To explore the role of miR-566 in glioblastoma, the expression levels of miR-566 (Fig. 1A) and VEGF mRNA (Fig. 1B) were evaluated by RT-qPCR in U87 cells post-lenti-AS-566 infection. The expression levels of miR-566 and VEGF mRNA were significantly decreased in the lenti-AS-566-infected cells, as compared with the untreated and lenti-NC-infected cells. These results indicate that lenti-AS-566 is able to effectively inhibit the expression of miR-566, and the mRNA expression of VEGF was downregulated when the expression of miR-566 was suppressed. 
A

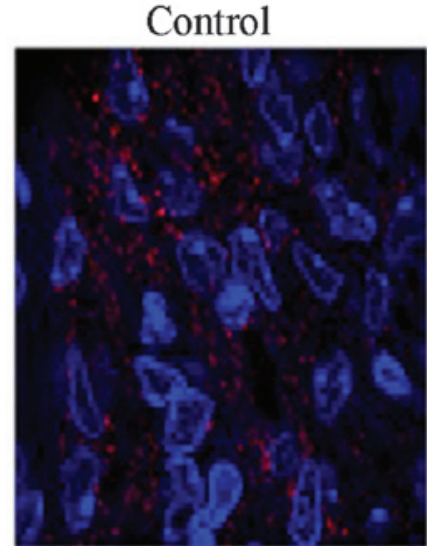

lenti-AS-566

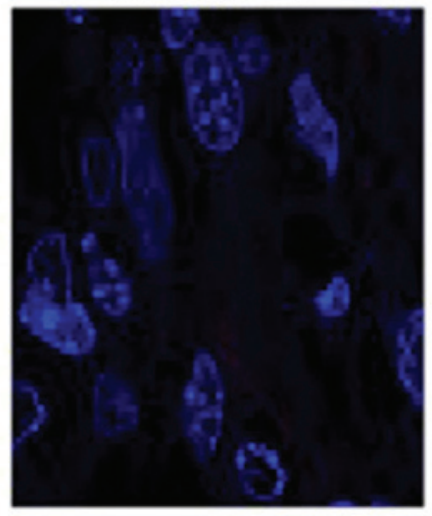

B

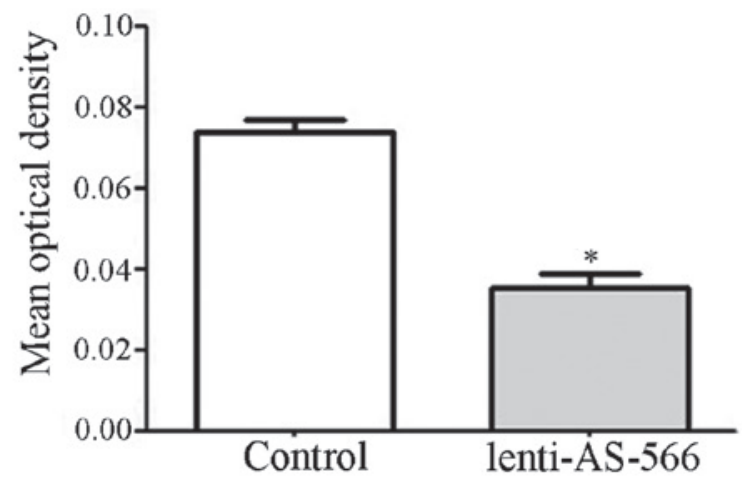

Figure 4. MicroRNA-566 (miR-566) was downregulated by lentivirus (lenti)-AS-miR-566 (566) in vivo. (A) The expression levels of miR-566 were significantly decreased in the lenti-AS-566 group, as compared with the control group (magnification, x200), as detected by fluorescence in situ hybridization. (B) Quantitative analysis of the fluorescence in situ hybridization results. Data are presented as the mean \pm standard deviation. " $\mathrm{P}<0.05$, as compared with the untreated control group.

A

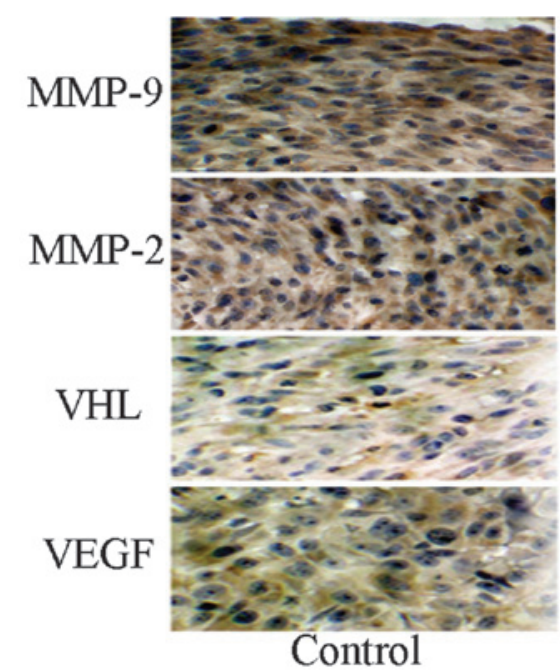

B

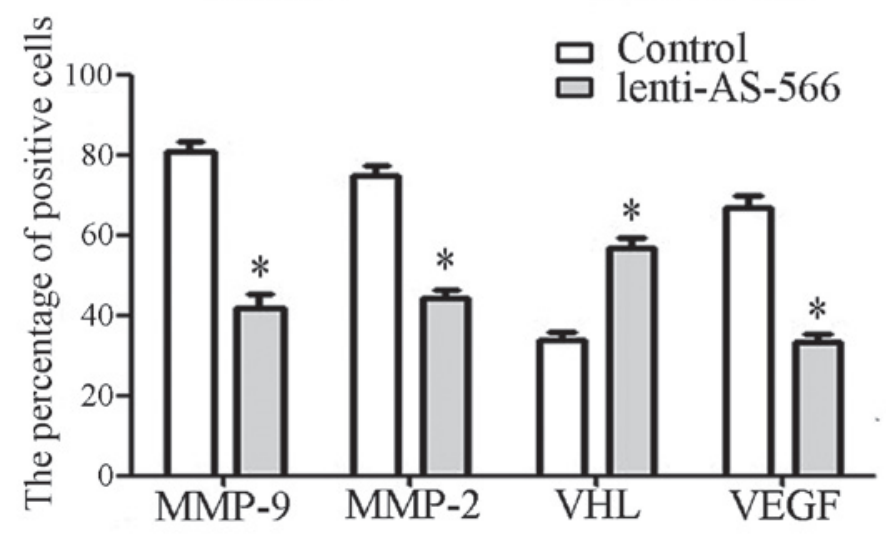

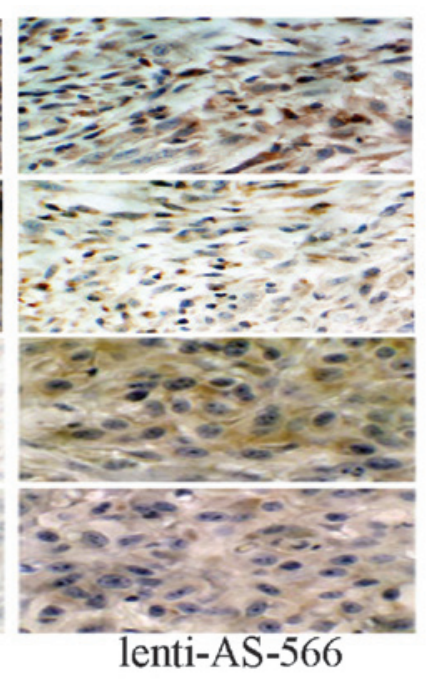

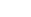


infected with lenti-NC (Fig. 1C). These results indicate that the VHL gene is a direct target of miR-566.

miR-566 regulates VEGF through the VHL/HIF1- $\alpha$ pathway in vitro. Western blot analysis was conducted to detect relative protein expression levels in the lenti-AS-566-infected U87 cells (Fig. 2A), and the results were quantitatively analyzed (Fig. 2B). The protein expression levels of VHL were increased in the U87 cells infected with lenti-AS-566, as compared with the untreated cells or those infected with lenti-NC. In addition, the protein expression levels of HIF-1 $\alpha$, MMP-2, MMP-9 and VEGF were decreased in the U87 cells infected with lenti-AS-566, as compared with the untreated cells or those infected with lenti-NC.

Upregulation of VHL inhibits the expression of VEGF. The relative protein expression levels were also measured following transfection of the U87 cells with a VHL expression plasmid (Fig. 3A), and the results were quantitatively analyzed (Fig. 3B). The protein expression levels of VHL were increased in the U87 cells transfected with the VHL expression plasmid, as compared with the untreated cells and those transfected with the VHL negative control (VHL-NC). The protein expression levels of HIF-1 $\alpha$ and VEGF were decreased in the U87 cells transfected with the VHL expression plasmid, as compared with the untreated cells and those transfected with VHL-NC. MMPs are associated with the invasion and migration of cells, and an increase in MMP-2 and MMP-9 is able to promote the invasion and migration of glioblastoma (26). In the present study, it was suggested that VHL was able to regulate the expression of VEGF via HIF-1 $\alpha$, and VHL was regulated by miR-566. Therefore, the inhibition of miR-566 may decrease the invasive and migratory abilities of the cells. These results indicate that miR-566 may modulate VEGF via targeting VHL in vitro.

miR-566 is downregulated in vivo. In order to further demonstrate that miR-566 was able to modulate VEGF by targeting VHL, an intracranial murine model was generated and brain tissue sections were obtained. The expression levels of miR-566 were measured in the tissue sections using fluorescence in situ hybridization (Fig. 4A), and the results were quantitatively analyzed (Fig. 4B). The expression levels of miR-566 were significantly decreased in the lenti-AS-566 group, as compared with the control group. These results indicate that lenti-AS-566 functioned effectively in the intracranial model, and was able to inhibit the expression of miR-566.

miR-566 modulates VEGF via targeting VHL in vivo. The expression levels of VHL and VEGF were detected in the brain tissue sections using immunohistochemistry (Fig. 5A), and the results were quantitatively analyzed (Fig. 5B). The expression levels of VHL in the tissue sections of the lenti-AS-566 group were increased, as compared with the control group. In addition, the expression levels of VEGF, MMP-2 and MMP-9 were decreased, as compared with the control group. These results indicate that VHL may regulate the expression of VEGF, and VHL is regulated by miR-566. Furthermore, the invasive and migratory abilities of the cells may be suppressed following the inhibition of miR-566, suggesting that miR-566 is able to modulate VEGF by targeting VHL in vivo.

\section{Discussion}

In recent years, numerous studies regarding miRNAs have been conducted, and miRNAs have been identified as having an important role in the initiation and progression of cancer, and have been suggested as a target for the treatment of glioblastoma. Kefas et al (10) transfected glioma cells with a miR-7 plasmid, which inhibited the activity of the epidermal growth factor receptor (EGFR) 3'-untranslated region (3'-UTR) by $83 \%$ and effectively reduced the invasive ability of the cells. Gabriely et al (27) specifically inhibited miR-21 in glioma, which reduced the invasiveness and malignancy of the cancer cells. In addition, Zhang et al (16) demonstrated that miR-566 was overexpressed in glioma cell lines, and inhibition of miR-566 was able to suppress the proliferative and invasive behavior of glioma cells via the EGFR/Akt pathway. Thus, miR-566 may function as an oncogene. The present study aimed to determine the role of miR-566 in glioblastoma, and to investigate the association between miR-566, VHL and VEGF.

The VHL gene is known to act as a tumor suppressor, and is also the causal gene of VHL disease (28). Furthermore, VHL is involved in the initiation and progression of numerous types of human cancer, particularly clear-cell renal cell carcinoma $(29,30)$. Chen et al transfected renal carcinoma cells that did not express pVHL with the normal VHL gene, and detected a marked inhibition in the growth of the tumor cells (31). In addition, a previous study demonstrated that VHL is able to target HIF-1 $\alpha$ for degradation by ubiquitin E3 ligase (23). It is well known that hypoxia is an important factor in the induction of VEGF, and under hypoxic conditions VEGF gene expression is upregulated due to induction of the HIF-1 $\alpha$ pathway (32). In the present study, U87 cells were infected with lenti-AS-566 and then incubated with VHL luciferase reporter vectors; VHL was identified as a direct target of miR-566. miR-566 may bind to the complimentary binding sites in the 3'-UTR of VHL (16). In addition, following inhibition of miR-566 by lenti-AS-566, western blotting revealed that VHL was upregulated, and HIF-1 $\alpha$, MMP-2, MMP-9 and VEGF were downregulated. Similar results were also obtained when the cells were transfected with a VHL expression plasmid. These results indicated that miR-566 was able to modulate VEGF by targeting VHL in vitro. In order to further demonstrate this regulatory relationship, an intracranial murine model was generated, and immunohistochemical staining demonstrated that VHL was upregulated, and MMP-2, MMP-9 and VEGF were downregulated when miR-566 was inhibited. These results suggested that miR-566 was able to modulate VEGF by targeting VHL in vivo, and the downregulation of MMP-2 and MMP-9 indicated that the invasive and migratory abilities of the cells were inhibited.

In conclusion, the present study demonstrated that miR-566 was able to modulate VEGF by targeting VHL in vitro and in vivo. In addition, the inhibition of miR-566 may suppress the invasion and migration of glioma; therefore, miR-566 may be considered a novel therapeutic target of glioblastoma. However, the mechanism underlying the effects of miR-566 in glioblastoma remains to be elucidated, and more in-depth research is required. 


\section{Acknowledgements}

The present study was supported by the China National Natural Scientific Fund (grant no. 81101916).

\section{References}

1. Krex D, Klink B, Hartmann C, von Deimling A, Pietsch T, Simon M, Sabel M, Steinbach JP, Heese O, Reifenberger G, Weller M and Schackert G; German Glioma Network: Long-term survival with glioblastoma multiforme. Brain 130: 2596-2606, 2007.

2. Stupp R, Mason WP, van den Bent MJ, Weller M, Fisher B, Taphoorn MJ, Belanger K, Brandes AA, Marosi C, Bogdahn U, et al; European Organisation for Research and Treatment of Cancer Brain Tumor and Radiotherapy Groups; National Cancer Institute of Canada Clinical Trial Group: Radiotherapy plus concomitant and adjuvant temozolomide for glioblastoma. N Engl J Med 352: 987-996, 2005.

3. Gabayan AJ, Green SB, Sanan A, Jenrette J, Schultz C, Papagikos M, Tatter SP, Patel A, Amin P, Lustig R, et al: GliaSite brachytherapy for treatment of recurrent malignant gliomas: A retrospective multi-institutional analysis. Neurosurgery 58: 701-709, 2006.

4. Giunti L, Pantaleo M, Sardi I, Provenzano A, Magi A, Cardellicchio S, Castiglione F, Tattini L, Novara F, Buccoliero AM, et al: Genome-wide copy number analysis in pediatric glioblastoma multiforme. Am J Cancer Res 4: 293-303, 2014.

5. Kirsch M, Schackert G and Black PM: Angiogenesis, metastasis, and endogenous inhibition. J Neurooncol 50: 173-180, 2000.

6. Carroll RS, Zhang J, Bello L, Melnick MB, Maruyama T and McL Black P: KDR activation in astrocytic neoplasms. Cancer 86: 1335-1341, 1999.

7. Oshika Y, Nakamura M, Tokunaga T, Ohnishi Y, Abe Y, Tsuchida T, Tomii Y, Kijima H, Yamazaki H, Ozeki Y, et al: Ribozyme approach to downregulate vascular endothelial growth factor (VEGF) 189 expression in non-small cell lung cancer (NSCLC). Eur J Cancer 36: 2390-2396, 2000.

8. Zhang X, Wu J and Gao D: Experimental research of gene therapy for human gliomas with vascular endothelial growth factor(165) antisense RNA. Zhonghua Yi Xue Za Zhi 80: 386-388, 2000 (In Chinese)

9. Drake CJ, LaRue A, Ferrara N and Little CD: VEGF regulates cell behavior during vasculogenesis. Dev Biol 224: 178-188, 2000.

10. Kefas B, Godlewski J, Comeau L, Li Y, Abounader R, Hawkinson M, Lee J, Fine H, Chiocca EA, Lawler S and Purow B: microRNA-7 inhibits the epidermal growth factor receptor and the Akt pathway and is down-regulated in glioblastoma. Cancer Res 68: 3566-3572, 2008.

11. Zhang Y, Dutta A and Abounader R: The role of microRNAs in glioma initiation and progression. Front Biosci (Landmark Ed) 17: 700-712, 2012.

12. Haapa-Paananen S, Chen P, Hellström K, Kohonen P, Hautaniemi S, Kallioniemi O and Perälä M: Functional profiling of precursor MicroRNAs identifies MicroRNAs essential for glioma proliferation. PLoS One 8: e60930, 2013.

13. Xiong J, Bing Z, Su Y, Deng D and Peng X: An integrated mRNA and microRNA expression signature for glioblastoma multiforme prognosis. PLoS One 9: e98419, 2014.

14. Chen L, Li H, Han L, Zhang K, Wang G, Wang Y, Liu Y, Zheng Y, Jiang T, Pu P, et al: Expression and function of miR-27b in human glioma. Oncol Rep. 26: 1617-1621, 2011.
15. Chen L, Zhang W, Yan W, Han L, Zhang K, Shi Z, Zhang J, Wang Y, Li Y, Yu S, et al: The putative tumor suppressor miR-524-5p directly targets Jagged-1 and Hes-1 in glioma. Carcinogenesis 33: 2276-2282, 2012

16. Zhang KL, Zhou X, Han L, Chen LY, Chen LC, Shi ZD, Yang M, Ren Y, Yang JX, Frank TS, et al: MicroRNA-566 activates EGFR signaling and its inhibition sensitizes glioblastoma cells to nimotuzumab. Mol Cancer 13: 63, 2014

17. Tanase CP, Enciu AM, Mihai S, Neagu AI, Calenic B and Cruceru ML: Anti-cancer therapies in high grade gliomas. Curr Proteomics 10: 246-260, 2013.

18. Odjélé A, Charest D and Morin P Jr: miRNAs as important drivers of glioblastomas: a no-brainer? Cancer Biomark 11: 245-252, 2012.

19. Gabriely G, Yi M, Narayan RS, Niers JM, Wurdinger T, Imitola J, Ligon KL, Kesari S, Esau C, Stephens RM, Tannous BA and Krichevsky AM: Human glioma growth is controlled by microRNA-10b. Cancer Res 71: 3563-3572, 2011.

20. Gaur AB, Holbeck SL, Colburn NH and Israel MA: Downregulation of Pdcd4 by mir-21 facilitates glioblastoma proliferation in vivo. Neuro Oncol 13: 580-590, 2011.

21. Zinnamosca L, Laudisi A, Petramala L, Marinelli C, Roselli M, Vitolo D, Montesani C and Letizia C: von Hippel Lindau disease with colon adenocarcinoma, renal cell carcinoma and adrenal pheochromocytoma. Intern Med 52: 1599-1603, 2013.

22. Chen L, Han L, Zhang K, Shi Z, Zhang J, Zhang A, Wang Y, Song Y, Li Y, Jiang T, et al: VHL regulates the effects of miR-23b on glioma survival and invasion via suppression of HIF-1 $\alpha /$ VEGF and $\beta$-catenin/Tcf-4 signaling. Neuro Oncol 14: 1026-1036, 2012.

23. Guo Y, Meng X, Ma J, Zheng Y, Wang Q, Wang Y and Shang H: Human papillomavirus 16 E6 contributes HIF-1 $\alpha$ induced warburg effect by attenuating the VHL-HIF-1 $\alpha$ interaction. Int J Mol Sci 15: 7974-7986, 2014

24. Kang CS, Pu PY, Li YH, Zhang ZY, Qiu MZ, Huang Q and Wang GX: An in vitro study on the suppressive effect of glioma cell growth induced by plasmid-based small interference RNA (siRNA) targeting human epidermal growth factor receptor. J Neurooncol 74: 267-273, 2005.

25. Kim JY, Grunke SD, Levites Y, Golde TE and Jankowsky JL: Intracerebroventricular viral injection of the neonatal mouse brain for persistent and widespread neuronal transduction. J Vis Exp 91: 51863, 2014.

26. Könnecke $\mathrm{H}$ and Bechmann I: The role of microglia and matrix metalloproteinases involvement in neuroinflammation and gliomas. Clin Dev Immunol 2013: 914104, 2013.

27. Gabriely G, Wurdinger T, Kesari S, Esau CC, Burchard J, Linsley PS and Krichevsky AM: MicroRNA 21 promotes glioma invasion by targeting matrix metalloproteinase regulators. Mol Cell Biol 28: 5369-5380, 2008

28. Ferrara N: Vascular endothelial growth factor: Molecular and biological aspects. Curr Top Microbiol Immunol 237: 1-30, 1999.

29. Baldewijns MM, van Vlodrop IJ, Vermeulen PB, Soetekouw PM, van Engeland M and de Bruïne AP: VHL and HIF signalling in renal cell carcinogenesis. J Pathol 221: 125-138, 2010.

30. Linehan WM, Rubin JS and Bottaro DP: VHL loss of function and its impact on oncogenic signaling networks in clear cell renal cell carcinoma. Int J Biochem Cell Biol 41: 753-756, 2009.

31. Chen F, Kishida T, Duh FM, Renbaum P, Orcutt ML, Schmidt L and Zbar B. Suppression of growth of renal carcinoma cells by the von Hippel-Lindau tumor suppressor gene. Cancer Res 55: 4804-4807, 1995.

32. Fang Y, Yu S, Ma Y, Sun P, Ma D, Ji C and Kong B: Association of Dll4/notch and HIF-1a-VEGF signaling in the angiogenesis of missed abortion. PloS One 8: e70667, 2013. 